\title{
USO DA MOXABUSTÃO E ACUPUNTURA EM GESTANTES COM APRESENTAÇÃO PÉLVICA: REVISÃO INTEGRATIVA
}

\author{
Mariana Haddad Rodrigues ${ }^{1}$, Vivian Inácio Zorzim²
}

\begin{abstract}
RESUMO: O objetivo deste estudo foi conduzir uma revisão integrativa a fim de identificar publicações sobre a moxabustão e acupuntura para versão de fetos em apresentação pélvica. A ferramenta de pesquisa Paciente, Intervenção, Comparação e Desfecho, disponibilizada pela base de dados da Biblioteca Nacional de Medicina dos Estados Unidos, foi utilizada para gerar uma lista de artigos. As palavras utilizadas para a busca foram os termos breech, moxibustion, acupuncture e cephalic. A busca foi realizada no mês de março de 2016. Foram encontrados 24 registros, 18 foram analisados, dentre os quais sete foram favoráveis ao uso da moxabustão, e cinco, desfavoráveis. A partir dos estudos realizados sobre o tema, há evidências de que a moxabustão seja segura e efetiva para tentar "corrigir" apresentações pélvicas, apresentando-se como uma ferramenta no complemento da assistência prestada por profissionais na área da obstetrícia.
\end{abstract}

DESCRITORES: Versão fetal; Artemísia; Enfermagem obstétrica; Pontos de acupuntura.

\section{USE OF MOXIBUSTION AND ACUPUNCTURE IN PREGNANT WOMEN WITH BREECH PRESENTATION: AN INTEGRATIVE REVIEW}

ABSTRACT: The objective in this study was to develop an integrative review to identify publications on moxibustion and acupuncture to turn fetuses in breech position. The research tool Patient, Intervention, Comparison and Outcome, available through the database of the United States National Library of Medicine, was used to produce a list of articles. The words used for the search were: breech, moxibustion, acupuncture and cephalic. The search was undertaken in March 2016. Twenty-four registers were found, 18 of which were analyzed, seven of them favorable to the use of moxibustion and five unfavorable. Based on the studies developed on the theme, there is evidence that moxibustion is safe and effective to try and "correct" breech presentations, as a tool to complement the care midwifery professionals provide.

DESCRIPTORS: Version, fetal; Artemisia vulgaris; Obstetric nursing; Acupuncture points.

\section{USO DE LA MOXIBUSTIÓN Y ACUPUNTURA EN EMBARAZADAS CON PRESENTACIÓN DE NALGAS: REVISIÓN INTEGRADORA}

RESUMEN: El objetivo de este estudio fue desarrollar una revisión integradora para identificar publicaciones sobre la moxibustión y acupuntura para versión de fetos en presentación de nalgas. La herramienta de investigación Paciente, Intervención, Comparación y Desfecho, publicada por la base de datos de la Biblioteca Nacional de Medicina de Estados Unidos, fue utilizada para generar un rol de artículos. Las palabras utilizadas para la búsqueda fueron: breech, moxibustion, acupuncture y cephalic. La búsqueda fue llevada a cabo en marzo del 2016. Fueron encontrados 24 registros, 18 fueron analizados, entre los cuales siete fueron favorables al uso de la moxibustión y cinco desfavorables. A partir de los estudios desarrollados sobre el tema, hay evidencias de que la moxibustión sea segura y efectiva para intentar "corregir" presentaciones de nalgas, siendo una herramienta para complementar la atención prestada por profesionales en el área de partería.

DESCRIPTORES: Versión fetal; Artemisia vulgaris; Enfermería obstétrica; Puntos de acupuntura.

${ }^{1}$ Enfermeira obstetra. Doutora em Enfermagem. Enfermeira da Prefeitura do Município de Itu. Itu, SP, Brasil.

${ }^{2}$ Enfermeira obstetra. Mestre em Enfermagem. Docente da Pós-Graduação em Enfermagem Obstétrica da Universidade Adventista de São Paulo. São Paulo, SP, Brasil. 


\section{- INTRODUÇÃO}

A incidência de fetos em apresentação pélvica no final da gestação varia entre $3-4 \%{ }^{(1)}$. As causas podem ser placenta prévia, gestação múltipla, anormalidade uterinas, baixo tônus uterino, oligodrânmio, polidrâmnio, tumores, fibromas, pelve contraída, cordão umbilical curto, restrição de crescimento intra-uterino e má-formações congênitas ${ }^{(2)}$.

O risco de mortalidade neonatal em partos pélvicos é de até 35 para mil nascidos vivos ${ }^{(3)}$. Verificouse que a mortalidade perinatal em partos vaginais pélvicos aumentou entre duas a cinco vezes comparada a partos cefálicos, e que a cirurgia cesariana diminuiu o risco de mortalidade neonatal, porém aumentou as chances de complicações maternas ${ }^{(4)}$.

No mundo, aproximadamente $11 \%$ das cirurgias cesarianas são realizadas por apresentação pélvica, podendo representar até $80 \%$ em alguns países ${ }^{(3)}$. As desvantagens da realização desta cirurgia são aumento de $29 \%$ no risco de mortalidade materna imediata, riscos de eventos adversos em gestações futuras, e risco para trombose, tromboembolismo pulmonar, infecção puerperal, hemorragia pós-parto, infecção urinária, infecção cirúrgica, ruptura uterina na próxima gestação e problemas respiratórios neonatais $^{(5)}$.

Existem fortes evidências e consenso suficientes (grau de recomendação A) para afirmar que a cesariana não é a opção de escolha para estes casos, e se necessária deverá ocorrer intraparto ${ }^{(3)}$. Antes de optar pela cirurgia, poderá ser tentada a versão cefálica externa (VCE) com 37 semanas ${ }^{(3)}$. A VCE é o procedimento pelo qual o médico obstetra tenta "corrigir" a apresentação pélvica em cefálica para que se siga o curso fisiológico da gestação e parto normal ${ }^{(6)}$. Usualmente, é realizada entre a $34^{\mathrm{a}}$ e $37^{\mathrm{a}}$ semanas gestacionais, com taxas de sucesso entre 50 e $60 \%{ }^{(5)}$, sendo uma opção antes de decidir pela cesariana, reduzindo em $55 \%$ o risco desta cirurgia ${ }^{(3)}$. Existem algumas contraindicações para sua realização, como o trabalho de parto ativo, cicatriz ou anormalidades uterinas e hemorragia vaginal, entre outras ${ }^{(6)}$. Contudo, este procedimento mostrou-se seguro e eficaz e deve ser oferecido às mulheres com apresentação pélvica em acompanhamento de pré-natal de risco habitual ${ }^{(3)}$.

As gestantes devem ser orientadas quanto aos benefícios e riscos da VCE, que incluem o desfecho mais desfavorável de morte perinatal, até complicações como circular de cordão, hipóxia fetal, rotura prematura de membranas ovulares e descolamento de placenta ${ }^{(7)}$ que podem levar a uma cesárea de emergência. No Brasil, a VCE não é popular entre os obstetras, por falta de preparo técnico para a realização do procedimento ${ }^{(3)}$.

Assim, a opção pela via de parto deverá ser baseada na decisão materna e experiência do obstetra; entretanto, em nosso país são poucos os profissionais que se sentem seguros para assistir partos pélvicos, por não possuírem treinamento e habilidades necessários para tanto ${ }^{(3)}$.

Existem outros métodos para induzir a "correção" de apresentação não-cefálica, tais como hipnose, exercícios posturais, quiropraxia, acupuntura, moxabustão ${ }^{(8)}$, ioga, homeopatia e estímulo acústico fetal $^{(7)}$. Há poucos estudos avaliando a eficácia desses métodos, sendo que a revisão sistemática da Biblioteca Cochrane recomenda a realização de mais estudos para determinar os efeitos e resultados da moxabustão e acupuntura para estes $\operatorname{casos}^{(1)}$.

A moxabustão, moxaterapia ou moxa, é uma técnica da medicina tradicional chinesa que consiste na estimulação de pontos de acupuntura por calor produzido pela queima da erva chamada Artemisia vulgaris $^{(9)}$, geralmente utilizada no formato de bastão que assemelha-se a um charuto. É uma técnica barata, segura, simples, que permite autoadministração, não invasiva, indolor e geralmente bem tolerada ${ }^{(10)}$. A moxabustão é contraindicada em casos de apresentação instável, gestações múltiplas, cesárea prévia ou cicatriz uterina, hemorragia pré-parto, diabetes, volume anormal de líquido amniótico, feto grande ou com má formação, desproporção céfalo-pélvica conhecida, história de infertilidade e de morte ou sofrimento fetal intra-útero ${ }^{(7)}$.

A moxabustão e acupuntura no ponto B67 $7^{(11)}$ (localizado no meridiano da Bexiga, próximo ao ângulo ungueal externo do $5^{\circ}$ dedo do pé) são indicadas para a versão cefálica de fetos em apresentação pélvica, e alguns estudos já foram conduzidos para avaliar a eficácia destes métodos ${ }^{(12)}$. Acredita-se que esta técnica estimule a produção de estrogênio placentário e prostaglandinas maternas, bem como 
promova contrações uterinas e movimentação fetal ${ }^{(13)}$.

O objetivo deste estudo é revisar a literatura a fim de identificar publicações sobre a moxabustão e acupuntura para versão de fetos em apresentação pélvica.

\section{- MÉTODO}

Foi realizada uma revisão integrativa da literatura, que tem por objetivo permitir que se formulem conclusões gerais sobre determinado tema, por meio da síntese de vários estudos publicados anteriormente ${ }^{(14)}$. Os resultados de uma revisão integrativa permitem gerar novas discussões e reflexões sobre a temática abordada, identificando lacunas de conhecimento que demandam novas investigações e realização de estudos futuros, além de pautar a tomada de decisão e melhoria da prática clínica ${ }^{(15)}$.

A revisão foi desenvolvida em etapas: (1) identificar o tema e estabelecer a questão de pesquisa; (2) estabelecer critérios de inclusão e exclusão de estudos e busca na literatura; (3) definir as informações a serem extraídas dos estudos selecionados e categorizar dos estudos; (4) avaliar os estudos incluídos na revisão integrativa; (5) interpretar os resultados; (6) apresentar a revisão e síntese do conhecimento ${ }^{(15)}$.

A questão de pesquisa utilizada foi: "Como a moxabustão e a acupuntura são utilizadas nas gestantes com feto em apresentação pélvica para que aconteça a versão cefálica?", elaborada conforme a estratégia Paciente, Intervenção, Comparação, Desfecho (PICO): anagrama em inglês para Patient, Intervention, Comparation e Outcome. A estratégia PICO auxilia a delimitar a busca, tornando-a mais objetiva, clara e específica, permitindo uma análise mais direcionada e completa, possibilitando conclusões de fácil identificação e aplicação(14-15).

O site da Biblioteca Nacional de Medicina dos Estados Unidos (PUBMED) disponibiliza uma ferramenta de pesquisa igualmente chamada $\mathrm{PICO}^{(16)}$, que permite inserir palavras-chave e descritores nas categorias de busca $\mathrm{P}$ - paciente/patient, I - intervenção/intervention, $\mathrm{C}$ - comparação/compare to, $\mathrm{O}$ - desfecho/outcome, gerando uma lista de artigos publicados. $\mathrm{O}$ item $\mathrm{C}$ não foi preenchido para não limitar a busca, permitindo que todas as comparações possíveis fossem incluídas.

Foram incluídos os artigos citados por esta lista, disponíveis na base de dados da PUBMED até o mês de fevereiro de 2016, contendo os descritores breech (posição pélvica), moxabustion (moxabustão), acupuncture (acupuntura), cephalic (posição cefálica). Foram excluídos os estudos em idioma não inglês e aqueles com indisponibilidade de acesso gratuito.

\section{RESUlTADOS}

Dos 24 artigos contidos na lista gerada pela plataforma PICO/PUBMED, seis foram excluídos da análise, resultando em 18 publicações, conforme demonstrado na Figura 1. 


\section{Número de registros identificados pela ferramenta \\ PICO/PUBMED $(\mathrm{n}=24)$}

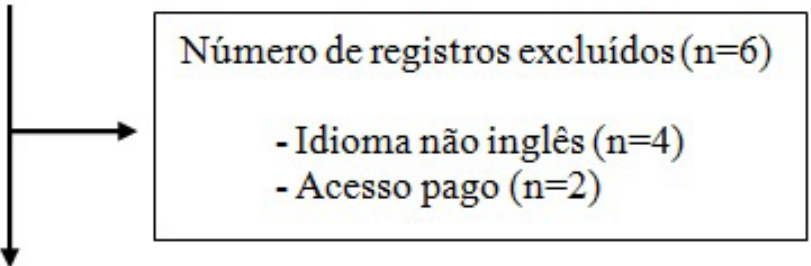

\section{- Ensaios clínicos $(\mathrm{n}=09)$ \\ - Revisões (n=04) \\ -Estudos quantitativos $(\mathrm{n}=04)$ \\ - Estudo qualitativo $(\mathrm{n}=01)$}

Número de registros incluídos na revisão $(\mathrm{n}=18)$

Figura 1 - Fluxograma das etapas empregadas na seleção da amostra. Itu, SP, Brasil, 2016

Todos os estudos consideraram como objetivo, ou desfecho primário, a "correção" da apresentação pélvica em gestações únicas e de baixo risco a partir do terceiro trimestre, utilizando a moxabustão no ponto B67.

Os artigos analisados estão caracterizados na Tabela 1 segundo autoria, periódico e ano de publicação, país de publicação, e delineamento/método.

Tabela 1 - Artigos analisados segundo autoria; periódico e ano de publicação; país de publicação; e tipo de estudo e método. Itu, SP, Brasil, 2016 (continua)

\begin{tabular}{|c|c|c|c|}
\hline Autoria & $\begin{array}{l}\text { Periódico e ano de } \\
\text { publicação }\end{array}$ & $\begin{array}{c}\text { País de } \\
\text { publicação }\end{array}$ & Delineamento / Método \\
\hline Bue L, Lauszus FF.(1) & $\begin{array}{l}\text { Dan Med J; } \\
\text { 2016;63(2). }\end{array}$ & Dinamarca & $\begin{array}{l}\text { Ensaio clínico randomizado } \\
\text { Cegamento: não }\end{array}$ \\
\hline Smith CA; Betts D. ${ }^{(17)}$ & $\begin{array}{l}\text { Complement Ther } \\
\text { Med; } 2014 ; 22(1): 75- \\
80 . \\
\end{array}$ & $\begin{array}{l}\text { Austrália e } \\
\text { Nova } \\
\text { Zelândia }\end{array}$ & $\begin{array}{l}\text { Estudo quantitativo } \\
\text { Consenso entre especialistas } \\
\text { Delphi. }\end{array}$ \\
\hline $\begin{array}{l}\text { Vas J, Aranda-Regules JM, Modesto } \\
\text { M, Ramos-Monserrat M, Barón M, } \\
\text { Aguilar I, et al.. }{ }^{(18)}\end{array}$ & $\begin{array}{l}\text { Acupunct Med; } \\
\text { 2013;31(1):31-8. }\end{array}$ & Espanha & $\begin{array}{l}\text { Ensaio clínico multicêntrico } \\
\text { Cegamento: avaliadores }\end{array}$ \\
\hline $\begin{array}{l}\text { Do CK, Smith CA, Dahlen H, Bisits A, } \\
\text { Schmied V. }{ }^{(12)}\end{array}$ & $\begin{array}{l}\text { BMC Complement } \\
\text { Altern Med; } \\
\text { 2011;(11):81. }\end{array}$ & Austrália & $\begin{array}{l}\text { Ensaio clínico randomizado } \\
\text { Cegamento: não }\end{array}$ \\
\hline $\begin{array}{l}\text { Van den Berg I, Kaandorp GC, Bosch } \\
\text { JL, Duvekot JJ, Arends LR, Hunink } \\
\text { MGM. }{ }^{(5)}\end{array}$ & $\begin{array}{c}\text { Complement Ther } \\
\text { Med; } 2010 ; 18(2): 67- \\
77 .\end{array}$ & Holanda & $\begin{array}{l}\text { Estudo quantitativo } \\
\text { Modelo hipotético }\end{array}$ \\
\hline $\begin{array}{l}\text { Guittier MJ, Pichon M, Dong H, Irion } \\
\text { O, Boulvain M. }{ }^{(19)}\end{array}$ & $\begin{array}{c}\text { Obstet Gynecol; } \\
\text { 2009; 114(5):1034-40. }\end{array}$ & Suiça & $\begin{array}{l}\text { Ensaio clínico randomizado } \\
\text { Cegamento: não }\end{array}$ \\
\hline Manyande A; Grabowska C. ${ }^{(2)}$ & $\begin{array}{c}\text { Midwifery; } \\
\text { 2009;25(6):774-80 }\end{array}$ & Reino Unido & Estudo quantitativo Prospectivo \\
\hline $\begin{array}{l}\text { Vas J, Aranda JM, Nishishinya B, } \\
\text { Mendez C, Martin MA, Pons J, et al. }{ }^{(13)}\end{array}$ & $\begin{array}{c}\text { Am J Obstet } \\
\text { Gynecol; } \\
2009 ; 201(3): 241-59 .\end{array}$ & Espanha & $\begin{array}{l}\text { Revisão sistemática e } \\
\text { Metanálise }\end{array}$ \\
\hline
\end{tabular}




\begin{tabular}{|c|c|c|c|}
\hline $\begin{array}{l}\text { Guittier MJ, Klein TJ, Dong H, } \\
\text { Andreoli N, Irion O, Boulvain M. }{ }^{(9)}\end{array}$ & $\begin{array}{l}\text { J Altern } \\
\text { Complement Med; } \\
2008 ; 14(10): 1231-3 .\end{array}$ & Suiça & $\begin{array}{l}\text { Ensaio clínico randomizado } \\
\text { Cegamento: avaliadores }\end{array}$ \\
\hline Mitchell M; Allen K. ${ }^{(20)}$ & $\begin{array}{c}\text { Complement } \\
\text { Ther Clin Pract; } \\
2008 ; 14(4): 264-72 \text {. }\end{array}$ & Reino Unido & $\begin{array}{l}\text { Pesquisa qualitativa } \\
\text { Abordagem fenomenológica }\end{array}$ \\
\hline $\begin{array}{l}\text { Vas J, Aranda JM, Barón M, Perea- } \\
\text { Milla E, Méndez C, Ramírez C, et al. }{ }^{(21)}\end{array}$ & $\begin{array}{l}\text { BMC Complement } \\
\text { Altern Med;2008; } \\
\text { 8:22. }\end{array}$ & Espanha & $\begin{array}{l}\text { Estudo quantitativo } \\
\text { Protocolo de ensaio clínico }\end{array}$ \\
\hline Cohain JS. ${ }^{(8)}$ & $\begin{array}{l}\text { Midwifery Today } \\
\text { Int Midwife; } \\
\text { 2007;(83):18-9, } 65 .\end{array}$ & $\begin{array}{c}\text { Não } \\
\text { informado }\end{array}$ & Revisão descritiva da literature \\
\hline $\begin{array}{l}\text { Cardini F, Lombardo P, Regalia AL, } \\
\text { Regaldo G, Zanini A, Negri MG, et } \\
\text { al. }{ }^{(22)}\end{array}$ & $\begin{array}{c}\text { BJOG; } \\
2005 ; 112(6): 743-7 .\end{array}$ & Itália & $\begin{array}{l}\text { Ensaio clínico multicêntrico } \\
\text { Cegamento: avaliadores }\end{array}$ \\
\hline Tiran D. ${ }^{(7)}$ & $\begin{array}{l}\text { Complement Ther } \\
\text { Nurs Midwifery; } \\
\text { 2004;10(4):233-8. }\end{array}$ & Reino Unido & Revisão descritiva da literatura \\
\hline $\begin{array}{l}\text { Neri I, Airola G, Contu G, Allais G, } \\
\text { Facchinetti F, Benedetto C. }{ }^{(23)}\end{array}$ & $\begin{array}{c}\text { J Matern Fetal } \\
\text { Neonatal Med; } \\
2004 ; 15(4): 247-52 \text {. }\end{array}$ & Itália & $\begin{array}{l}\text { Ensaio clínico multicêntrico } \\
\text { Cegamento: avaliadores }\end{array}$ \\
\hline $\begin{array}{l}\text { Neri I, Fazzio M, Menghini S, Volpe } \\
\text { A, Facchinetti F. }\end{array}$ & $\begin{array}{c}\text { J Soc Gynecol } \\
\text { Investig; } \\
2002 ; 9(3): 158-62 \text {. }\end{array}$ & Itália & $\begin{array}{l}\text { Ensaio clínico randomizado } \\
\text { Cegamento: avaliadores }\end{array}$ \\
\hline Ewies A; Olah K.(10) & $\begin{array}{l}\text { Acupunct Med; } \\
2002 ; 20(1): 26-9 .\end{array}$ & $\begin{array}{c}\text { Não } \\
\text { informado }\end{array}$ & Revisão descritiva da literatura \\
\hline Cardini F; Weixin H. ${ }^{(25)}$ & $\begin{array}{c}\text { JAMA; } \\
1998 ; 280(18): 1580-4 .\end{array}$ & China & $\begin{array}{l}\text { Ensaio clínico randomizado } \\
\text { Cegamento: avaliadores. }\end{array}$ \\
\hline
\end{tabular}

Nos ensaios clínicos ${ }^{(1,9,12,18-19,22-25)}$, foram excluídas as mulheres com gestações gemelares, risco de parto prematuro, comorbidades renais ou cardíacas, placenta prévia, historia de hemorragia pré-parto, restrição de crescimento intra-uterino, doença hipertensiva, isoimunização, cirurgia uterina prévia, anomalias uterinas, alterações fetais congênitas, contra-indicações para o parto vaginal e óbito fetal intra-útero.

Os estudos foram conduzidos em diferentes países, como Dinamarca ${ }^{(1)}$, Austrália( ${ }^{(12,17)}$ e Nova Zelândia $^{(17)}$, Espanha ${ }^{(13,18,21)}$, Holanda ${ }^{(5)}$, Suíça ${ }^{(9,19)}$, Reino Unido ${ }^{(2,7,20)}$, Itália ${ }^{(22-24)}$ e China ${ }^{(25)}$, demonstrando que, apesar da técnica ser originalmente chinesa, já está bem popularizada nos países ocidentais. Percebe-se que muitos profissionais estão tão familiarizados com a moxabustão a ponto de sentir a necessidade de testar a sua eficácia para melhor segurança e confiança em aplicá-la na prática clínica.

A Tabela 2 apresenta os artigos analisados segundo amostra e idade gestacional (IG); intervenção; e conclusão.

Tabela 2 - Artigos analisados segundo amostra e IG; intervenção; e conclusão. Itu, SP, Brasil, 2016 (continua)

\begin{tabular}{|c|c|c|}
\hline Amostra e IG & Intervenção & Conclusão \\
\hline $\begin{array}{l}N=200 \\
I G: 33 s\end{array}$ & a) $M(n=102)$, b) $C(n=98)$ & Sem evidências de que $M$ tenha efeito ${ }^{(1)}$. \\
\hline $\mathrm{N}=16$ & Questionário online para experts. & $\begin{array}{l}\text { O estudo possui validade clínica para criar um } \\
\text { protocolo de estudo }{ }^{(17)} \text {. }\end{array}$ \\
\hline $\begin{array}{l}N=406 \\
I G: 33-35 s\end{array}$ & $\begin{array}{l}\text { a) } M(n=136), \text { b) } M P(n=136) \text {, } \\
\text { b) } C+E P(n=134)\end{array}$ & $\begin{array}{l}\text { M é eficaz e segura e deve ser considerada } \\
\text { como opção de intervenção }{ }^{(18)} \text {. }\end{array}$ \\
\hline $\begin{array}{l}N=20 \\
I G: 34-36.6 s\end{array}$ & a) $M(n=10), C(n=10)$ & $\begin{array}{l}\text { Estudo sem poder estatístico devido amostra } \\
\text { pequena }{ }^{(12)} \text {. }\end{array}$ \\
\hline
\end{tabular}




\begin{tabular}{|c|c|c|}
\hline $\begin{array}{l}\mathrm{N}=\text { não se aplica } \\
\text { IG: } 33 \mathrm{~s}\end{array}$ & $\begin{array}{l}\text { a) } M \\
\text { b) } C\end{array}$ & $\begin{array}{l}\text { M apresentou redução de apresentações } \\
\text { pélvicas e cesáreas, mais econômico que } C \text {, } \\
\text { incluindo a } \mathrm{VCE}^{(5)} \text {. }\end{array}$ \\
\hline $\begin{array}{l}N=212 \\
I G: 34-36 s\end{array}$ & $\begin{array}{l}\text { a) } M(n=106) \\
\text { b) } C(n=106)\end{array}$ & $\begin{array}{l}\text { Não demonstrou benefícios da M para } \\
\text { "correção" de posição pélvica }{ }^{(19)} \text {. }\end{array}$ \\
\hline $\begin{array}{l}N=76 \\
\mathrm{IG}: 28 \mathrm{~s}\end{array}$ & $M(n=76)$ & $\begin{array}{l}\text { Há evidências para acreditar que } M \text { pode } \\
\text { reduzir o número de apresentações pélvicas }{ }^{(2)} \text {. }\end{array}$ \\
\hline $\begin{array}{l}\mathrm{N}=1087 \\
\mathrm{IG}: \geq 32 \mathrm{~s}\end{array}$ & a) $M$, b) $C$, c) $E P$, d) $A$ & $\begin{array}{l}\text { M (isolada ou combinada a EP) produziu efeito } \\
\text { positivo sobre C ou somente } E P^{(13)} \text {. }\end{array}$ \\
\hline $\begin{array}{l}N=65 \\
I G: 34-36 s\end{array}$ & CTB antes, durante, após (10`/20`/10`) M. & $\begin{array}{l}\text { M não causou alterações no bem estar fetal e } \\
\text { materno ou efeitos adversos }{ }^{(9)} \text {. }\end{array}$ \\
\hline $\begin{array}{l}N=8 \\
I G: \geq 33 s\end{array}$ & M e entrevista. & $\begin{array}{l}\text { Boa aceitação e adesão da técnica, sem efeitos } \\
\text { colaterais }^{(20)} \text {. }\end{array}$ \\
\hline $\begin{array}{l}\mathrm{N}=492 \\
\mathrm{IG}: 33-35 \mathrm{~s}\end{array}$ & a) $M$, b) $M P$, c) $C+E P$ & Não se aplica ${ }^{(21)}$ \\
\hline Não se aplica. & $\begin{array}{l}\text { a) VCE, b) M ou } A, \text { c) quiropraxia, d) } \\
\text { hipnoterapia, e) exercícios posturais. }\end{array}$ & $\begin{array}{l}\text { Não há evidências suficientes para recomendar } \\
\text { absolutamente qualquer dos } 5 \text { métodos } \\
\text { mencionados }^{(8)} \text {. }\end{array}$ \\
\hline $\begin{array}{l}\mathrm{N}=123 \\
\mathrm{IG}: 32-33.3 \mathrm{~s}\end{array}$ & a) $M(n=65)$, b) $C(n=58)$ & $\begin{array}{l}\text { Falhas no protocolo e amostra insuficiente } \\
\text { levaram à interrupção do estudo(2). }\end{array}$ \\
\hline Não se aplica. & $\begin{array}{l}\text { Descrição de técnicas: } \\
\text { a) M, b) pasta de gengibre, c) homeopatia, } \\
\text { d) estímulo acústico fetal, e) hipnose, f) } \\
\text { quiropraxia, g) ioga, h) outras. }\end{array}$ & $\begin{array}{l}\text { Parteiras são os profissionais com melhor } \\
\text { oportunidade de fornecer informações sobre } \\
\text { métodos alternativos para "correção" de } \\
\text { apresentação pélvica(7). }\end{array}$ \\
\hline $\begin{array}{l}\mathrm{N}=226 \\
\mathrm{IG}: 33-35 s\end{array}$ & a) $M$ e $A(n=112)$, b) $C(n=114)$ & M e A mais eficazes que $C^{(23)}$ \\
\hline $\begin{array}{l}\mathrm{N}=12 \\
\mathrm{IG}: 33 \mathrm{~s}\end{array}$ & $\begin{array}{l}\text { a) AP, b) A, CTB antes e após (20’ 20’) } \\
\text { ambos. }\end{array}$ & $\begin{array}{l}\text { Alterações na FCF e MF durante } A \text {, e ausentes } \\
\text { em } \mathrm{AP}^{(24)} \text {. }\end{array}$ \\
\hline Não se aplica. & Relata características da técnica de moxa. & Sem evidências de que $M$ tenha efeito ${ }^{(10)}$. \\
\hline $\begin{array}{l}\mathrm{N}=130 \\
\mathrm{IG}: 33 \mathrm{~s}\end{array}$ & a) $M(n=130)$, b) $C(n=130)$ & $\begin{array}{l}\text { M foi efetiva para induzir aumento de versões } \\
\text { cefálicas nessa população }{ }^{(25)} \text {. }\end{array}$ \\
\hline
\end{tabular}

Legenda: $\mathrm{N}=$ número, $\mathrm{IG}=$ idade gestacional, $\mathrm{M}=$ moxa, $\mathrm{MP}=$ moxa placebo, $\mathrm{C}=$ cuidado usual, $\mathrm{EP}=$ exercícios posturais, $\mathrm{CTB}=$ cardiotocografia fetal basal, VCE = versão cefálica externa, $\mathrm{A}=$ acupuntura, $\mathrm{AP}=$ acupuntura placebo, $\mathrm{FCF}=\mathrm{frequência}$ cardíaca fetal, MF = movimentação fetal.

A maioria dos estudos coletou dados sociodemográficos ${ }^{(1,9,12,18-19,22-25)}$, tipos de parto ${ }^{(1-2,5,9,12-13,18-20,22-25)}$, frequência de $V C E^{(1,9,12,18-19,22-25)}$, efeitos adversos e sinais e sintomas ${ }^{(1,9,12,18-20,22-24)}$, relatados durante $o$ tratamento. Alguns estudos se propuseram a identificar a opinião dos profissionais envolvidos quanto à técnica aplicada ${ }^{(5,17,20)}$.

Todos os estudos intervencionistas ${ }^{(1-2,9,12-13,18-19,21-25)}$ aplicaram a técnica a partir do $3^{\circ}$ trimestre de gestação, principalmente a partir da $33^{\mathrm{a}}$ semana, e caso a versão cefálica não ocorresse, a VCE era oferecida.

As participantes relataram alguns efeitos adversos advindos da moxaterapia como mau cheiro(1,22), cefaleia $^{(1)}$, náuseas ${ }^{(1,18,20,22)}$, tontura ${ }^{(18)}$, hipertensão leve ${ }^{(18)}$, dor abdominal ${ }^{(18,22)}$, dor de $\operatorname{garganta}^{(10,22)} \mathrm{e}$ queimaduras $^{(10)}$. Os sinais e sintomas vivenciados foram contração uterina ${ }^{(1,9,12,18-19,20,24-25)}$, movimentação fetal $\left.\right|^{(1,19-20,24)}$, soluço fetal ${ }^{(18)}$, relaxamento ${ }^{(9,20)}$, urgência urinária ${ }^{(20)}$ e aumento do peristaltismo intestinal ${ }^{(20)}$.

Houve boa aceitação e aderência ao tratamento com a moxabustão por parte das gestantes, embora as participantes de um estudo relatassem desconfortos com frequência e muitas desistiram do tratamento por este motivo, impossibilitando a conclusão do mesmo ${ }^{(21)}$.

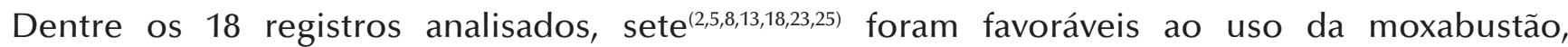
identificando a eficácia do método quando comparada ao cuidado usual ou conduta expectante; enquanto que cinco tiveram resultados desfavoráveis ${ }^{(1,10,12,19,22)}$. Os outros seis estudos não afirmaram 
categoricamente seu posicionamento contra ou a favor da técnica, pois se tratavam de descrição de um protocolo de estudo ${ }^{(17,21)}$, ensaio clínico sobre os efeitos da moxa na vitalidade fetal ${ }^{(9,24)}$, estudo qualitativo sobre a opinião das participantes sobre a moxa e apresentação pélvica ${ }^{(20)}$, revisão narrativa sobre as técnicas para "correção" da apresentação pélvica ${ }^{(7)}$.

Um grupo de pesquisadores espanhóis desenvolveu um protocolo de ensaio clínico ${ }^{(21)}$ que foi colocado em prática ${ }^{(18)}$.

\section{DISCUSSÃO}

\section{Das variáveis maternas e fatores associados à moxabustão}

Quanto à paridade, um ensaio clínico inglês identificou maiores chances de sucesso na versão cefálica entre multíparas quando comparadas às primíparas (IC 95\% 0,05-0,94, p<0,05), provavelmente porque seus músculos abdominais podem estar mais flexíveis ${ }^{(2)}$. As chances duplicaram quando terceiros (família e amigos) participaram da administração da técnica (IC 95\% 0,87-5,16, p<0,04), sugerindo que a motivação foi tão intensa a ponto de envolver um terceiro e favorecer que as instruções fossem seguidas meticulosamente ${ }^{(2)}$. Consequentemente, a gestante recebeu mais atenção e apoio, permitindo que se sentisse mais segura e relaxada ${ }^{(2)}$.

Já os resultados de estudo italiano ${ }^{(22)}$ apontaram para desfechos mais favoráveis quanto à versão cefálica com 35 semanas entre mulheres com idade $\leq 31$ anos, $n=25(45 \%)$ quando comparadas àquelas $\geq 31$ anos, $\mathrm{n}=17(27 \%)$. Quanto à escolaridade, o desfecho foi mais favorável àquelas com $\leq 8$ anos escolares, $n=14(47 \%)$ comparadas àquelas com nível médio ou superior, $n=26(29 \%)^{(22)}$.

\section{A moxaterapia é segura para gestantes e fetos}

Não houve evidências que associem a moxabustão ao risco de parto prematuro, portanto pesquisadores espanhóis sugerem que quanto mais cedo o tratamento for iniciado, melhores são as chances de sucesso ${ }^{(18)}$. O mesmo estudo não identificou alterações clínicas no ritmo cardíaco materno ou fetal durante as sessões de moxabustão, a que foram submetidas os dois grupos (moxa e moxa placebo) de mulheres quando comparadas àquelas que receberam apenas o cuidado usual ${ }^{(18)}$. Não foi relatado nenhum caso de hiper/hipotensão materna durante as sessões com moxa ${ }^{(9)}$ e houve significância estatística na redução do uso de ocitocina no grupo submetido à técnica, comparado ao grupo submetido ao cuidado usual ${ }^{(13)}$.

Em metanálise, as variáveis de taxa de cesárea, Apgar $<7$ no $5^{\circ}$ minuto, parto instrumental e ruptura prematura de membranas mostraram tendência a favor do grupo que utilizou a moxabustão, porém sem relevância estatística, devido à heterogeneidade dos estudos ${ }^{(13)}$.

Para verificar a segurança do bem estar fetal, estudo conduzido na Suíça mensurou os efeitos adversos da técnica ao utilizar a cardiotocografia antes, durante e após as sessões, que foram avaliadas por três observadores independentes utilizando o escore de Fischer ${ }^{(9)}$. Este foi o primeiro estudo a avaliar sistematicamente a segurança fetal através da cardiotocografia, identificando pequena redução na FCF e no número de acelerações durante e logo após as sessões, ambas associadas à menor frequência de movimentos fetais dentro da normalidade ${ }^{(9)}$. Apesar do mecanismo de ação da acupuntura e moxabustão no ponto B67 ainda ser desconhecido, existe uma mudança no padrão de movimentação fetal e ritmo cardíaco durante a aplicação da técnica ${ }^{(23)}$.

\section{Adesão ao tratamento e efeitos durante o uso da moxabustão}

A maioria (10/12) das participantes definiu o tratamento como favorável ou muito favorável, corroborando com a aderência ao tratamento, pois nenhuma participante faltou às sessões ou desistiu do protocolo de estudo ${ }^{(9)}$. Algumas participantes relataram ter contrações uterinas e aumento de movimentação fetal durante o dia de tratamento; nenhuma relatou efeitos adversos, e outras consideraram o tratamento agradável e relaxante ${ }^{(9)}$. Mais de $98 \%$ das participantes recomendariam a 
técnica para uma amiga na mesma situação e 95\% fariam de novo, se necessário(19).

Alguns desconfortos foram relatados durante a aplicação da técnica, como por exemplo, a sensação de dor, que foi avaliada utilizando uma escala visual analógica ( 0 a 100), e variou entre 0 e $15^{(9)}$. Em estudo italiano, $41 \%$ das gestantes incluídas no grupo com moxa relataram náuseas, dor de garganta e contrações dolorosas, sendo que $21 \%$ desistiram do tratamento por esses motivos ${ }^{(22)}$. A dificuldade na adesão ao tratamento poderia ser explicada por fatores culturais, pois o estudo em questão foi conduzido na Itália ${ }^{(22)}$, onde a prática não é tão comum quanto na China, onde o estudo modelo foi conduzido ${ }^{(25)}$.

Entre as mulheres que mais relataram efeitos adversos, houve menor sucesso na versão cefálica, hipoteticamente, porque os sintomas desagradáveis impediram o relaxamento dos músculos abdominais e dificultando assim a versão espontânea ${ }^{(2)}$.

\section{Métodos alternativos para versão cefálica}

Em busca simples pela Internet, há citações de outros métodos alternativos como tocar música sobre o abdome, aumentando o relaxamento e levando a uma versão espontânea ${ }^{(7)}$. Neste sentido, foi verificado, por meio de encefalografia magnética, que pulsos sonoros repetidos provocam reações fetais $^{(7)}$. Também verificou-se mudança no posição da linha média dorsal do feto, de anterior para lateral, o que aumenta as chances de sucesso de $\mathrm{VCE}^{(7)}$.

Quanto ao uso da acupuntura, um pequeno número de especialistas recomendou o uso da técnica, a ser administrada 2-3 vezes por semana, podendo ser utilizados outros pontos além do B67, como o BP6 ${ }^{(11)}, V G 20^{(11)}$, E36 ${ }^{(11)}$ e VB34(11); e não houve concordância de opiniões sobre a necessidade de obtenção

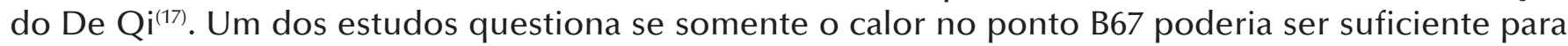
produzir os mesmos resultados que a moxabustão, propondo um estudo para comparar a técnica com outra fonte de calor que não a moxa(18).

A Medicina Tradicional Chinesa também indica o uso de uma pasta feita com gengibre fresco moído a ser aplicado durante a noite, por 10 dias, como outra fonte de calor a ser utilizada no ponto $\mathrm{B} 67^{(7)}$. Existem poucos estudos sobre a técnica, com taxas de sucesso de versão cefálica espontânea de até $80 \%$ e nenhum deles relata efeitos adversos ${ }^{(7)}$.

A homeopatia também está indicada para casos de apresentação pélvica, recomendando o uso das fórmulas Pulsatilla e Natrum muriaticum ${ }^{(7)}$.

Profissionais australianos na área de obstetrícia (médicos e parteiras) expressaram opiniões positivas sobre o uso de terapias alternativas e complementares em geral, apontando que há espaço para estas técnicas no cuidado em saúde habitual ${ }^{(12)}$. Demonstram a percepção de que existem lacunas sobre a eficácia clínica da técnica, e sugerem o senso de responsabilidade de garantir novos tratamentos que sejam seguros e efetivos ${ }^{(12)}$.

\section{Implicações, benefícios e reflexão da prática}

Do ponto de vista dos gerentes e profissionais de serviços de pré-natal, para que a técnica seja disponibilizada no serviço público, sua eficácia e custo-benefício deverão ser comprovados com evidências ${ }^{(20)}$. Houve um consenso de que parteiras podem aplicar a técnica desde que haja protocolo para tanto; e o encaminhamento, quando do diagnóstico de apresentação pélvica, deverá ser feito antes de 33 semanas $^{(20)}$.

Autores holandeses afirmam que mesmo não havendo garantia de sucesso no uso desta técnica; a mera tentativa pode ser benéfica em países de baixa renda ou em áreas rurais distantes de centros médicos, pois as mulheres que habitam esses lugares têm menor probabilidade de acesso a uma VCE ou cirurgia cesariana em casos de apresentação pélvica ${ }^{(5)}$.

O diagnóstico de apresentação pélvica gera ansiedade e preocupação, principalmente sobre as consequências maternas e fetais de um parto pélvico ou uma cirurgia cesariana, bem como frustração 
por não ter a oportunidade de vivenciar um parto normal e apreensão com a opção de VCE, mesmo que esta fosse a única opção para tentar um parto vaginal ${ }^{(20)}$. As gestantes relataram que a VCE pode ser invasiva ao "forçar" o bebê a virar, e que poderia causar problemas futuros ${ }^{(20)}$. Conhecendo as implicações de um parto pélvico ou uma cesárea, as mulheres demonstraram contentamento em aceitar a oferta de tratamento com a moxabustão(20).

Em relação aos sentimentos quanto ao tipo de parto, no momento da randomização, metade das mulheres (106/212) declarou ter preferência pelo parto vaginal, enquanto que, de fato, menos de um quarto delas optou por ele ${ }^{(19)}$. Outro dado importante foi que nem todas as gestantes submetidas à moxabustão tentou a VCE $(60 / 106)^{(19)}$. Os autores tecem duas hipóteses para explicá-lo: relutância em submeter-se a uma cesárea, porém aceitando-a como realidade após uma tentativa não sucedida de "correção" da apresentação pelo uso de um método alternativo, independente de qual tenha sido. Ou a tomada de decisão após conversa com o obstetra sobre as alternativas, no final da gestação, ao tomar ciência dos resultados de um ensaio clínico anterior ${ }^{(19)}$.

É importante que mulheres que procuram alternativas para a apresentação pélvica sejam atendidas por um profissional capaz de fornecer uma gama de opções, tanto complementares quanto convencionais, e que seja capaz de avaliar apropriadamente as indicações e contraindicações de cada método $^{(7)}$. Profissionais que praticam a Medicina Tradicional Chinesa ou homeopatia e não são da área da obstetrícia serão incapazes de confirmar a apresentação pélvica antes de aplicar um método específico, e desconhecem os fatores de risco, contraindicações, e complicações obstétricos ${ }^{(7)}$. Este argumento per se justifica a necessidade de treinar parteiras para oferecer estas alternativas para "correção" de apresentação pélvica, e este conteúdo deveria fazer parte da educação continuada destes profissionais ${ }^{(7)}$.

\section{CONSIDERAÇÕES FINAIS}

Os resultados encontrados nos estudos acima divergem quanto à eficácia da moxabustão como técnica para versão cefálica de fetos pélvicos. São necessários dados mais sólidos, com pesquisas que incluam o mesmo protocolo de início de idade gestacional, tempo de aplicação da técnica e frequência para afirmar de que forma ela pode ser utilizada com maior percentual de sucesso.

Contudo, alguns resultados nos permitem afirmar que é uma técnica segura, efetiva, simples, não invasiva e de baixo custo que pode ser oferecida para mulheres com gestação em apresentação pélvica antes de indicar a VCE, que é um procedimento mais caro e com riscos em potencial. Ao ser oferecida como alternativa, antes da VCE ou juntamente a esta, poderá aumentar as chances de redução do número de cirurgias cesarianas, seus custos, e suas taxas de morbimortalidade fetal e materna.

Profissionais da obstetrícia devem fornecer às gestantes informações imparciais sobre os métodos convencionais e complementares para "correção" da apresentação pélvica, incluindo seus riscos e benefícios. Independente da decisão tomada pela gestante, o profissional deverá acolher e apoiála, quando possível aplicando as técnicas escolhidas ou referenciando-a a serviços ou profissionais competentes e capacitados para efetivá-las (moxaterapia, VCE, parto pélvico, cirurgia cesariana).

\section{REFERÊNCIAS}

1. Bue L, Lauszus FF. Moxibustion did not have an effect in a randomised clinical trial for version of breech position. Dan Med J. [Internet] 2016;63(2) [acesso em 03 jan 2016]. Disponível: http://www.danmedj.dk/portal/ page/portal/danmedj.dk/dmj_forside/PAST_ISSUE/2016/DMJ_2016_02/A5199.

2. Manyande A, Grabowska C. Factors affecting the success of moxibustion in the management of a breech presentation as a preliminary treatment to external cephalic version. Midwifery. [Internet] 2009;25(6) [acesso em 03 jan 2016]. Disponível: http://dx.doi.org/10.1016/j.midw.2008.08.003.

3. Amorim MMR, Souza ASR, Porto AMF. Indicações de cesariana baseadas em evidências: parte I. Femina. [Internet] 2010;38(8) [acesso em 03 jan 2016]. Disponível: http://www.febrasgo.org.br/site/wp-content/ uploads/2013/05/Femina-v38n8_pg415-422.pdf. 
4. Hofmeyr GJ, Hannah ME, Lawrie TA. Planned caesarean section for term breech delivery. Cochrane Database Syst Rev. [Internet] 2015;(7) [acesso em 12 jan 2016]. Disponível: http://dx.doi.org/10.1002/14651858.CD000166. pub2.

5. Van den Berg I, Kaandorp GC, Bosch JL, Duvekot JJ, Arends LR, Hunink MGM. Cost-effectiveness of breech version by acupuncture-type interventions on BL 67, including moxibustion, for women with a breech foetus at 33 weeks gestation: a modelling approach. Complement Ther Med. [Internet] 2010;18(2) [acesso em 03 jan 2016]. Disponível: http://dx.doi.org/10.1016/j.ctim.2010.01.003.

6. Haddad SMT, Cececatti JG. Strategies directed to professionals for reducing unnecessary cesarean sections in Brazil. Rev. Bras. Ginecol. Obstet. [Internet] 2011;33(5) [acesso em 03 jan 2016]. Disponível: http://dx.doi. org/10.1590/S0100-72032011000500008.

7. Tiran D. Breech presentation: increasing maternal choice. Complement Ther Nurs Midwifery. [Internet] 2004;10(4) [acesso em 05 jan 2016]. Disponível: http://dx.doi.org/10.1016/j.ctnm.2004.01.005.

8. Cohain JS. Turning breech babies after 34 weeks: the if, how, \& when of turning breech babies. Midwifery Today Int Midwife. [Internet] 2007;(83) [acesso em 05 jan 2016]. Disponível: http://europepmc.org/abstract/ $\mathrm{med} / 17927112$.

9. Guittier MJ, Klein TJ, Dong H, Andreoli N, Irion O, Boulvain M. Side-effects of moxibustion for cephalic version of breech presentation. J Altern Complement Med. [Internet] 2008;14(10) [acesso em 05 jan 2016]. Disponível: http://dx.doi.org/10.1089/acm.2008.0134.

10. Ewies A, Olah K. Moxibustion in breech version-a descriptive review. Acupunct Med. [Internet] 2002;20(1) [acesso em 05 jan 2016]. Disponível: http://aim.bmj.com/content/20/1/26.long.

11. Yamamura Y. Acupuntura Tradicional: a arte de inserir. $2^{a}$ ed. São Paulo: Roca; 2004.

12. Do CK, Smith CA, Dahlen H, Bisits A, Schmied V. Moxibustion for cephalic version: a feasibility randomised controlled trial. BMC Complement Altern Med. [Internet] 2011;(11) [acesso em 05 jan 2016]. Disponível: http:// dx.doi.org/10.1186/1472-6882-11-81.

13. Vas J, Aranda JM, Nishishinya B, Mendez C, Martin MA, Pons J, et al. Correction of nonvertex presentation with moxibustion: a systematic review and metaanalysis. Am J Obstet Gynecol. [Internet] 2009;201(3) [acesso em 05 jan 2016]. Disponível: http://dx.doi.org/10.1016/j.ajog.2008.12.026.

14. Schneider F, Pedrolo E, Lind J, Schwanke AA, Danski MTR. Prevenção e tratamento de radiodermatite: uma revisão integrativa. Cogitare Enferm. [Internet] 2013;18(3) [acesso em 13 set 2016]. Disponível: http://dx.doi. org/10.5380/ce.v18i3.33575.

15. Mendes KDS, Silveira RCCP, Galvão CM. Revisão integrativa: método de pesquisa para a incorporação de evidências na saúde e na enfermagem. Texto Contexto Enferm. [Internet] 2008;17(4) [acesso em 13 set 2016]. Disponível: https://dx.doi.org/10.1590/S0104-07072008000400018.

16. Biblioteca Nacional de Medicina dos Estados Unidos (PUBMED). [Internet] Search MEDLINE/PubMed via PICO with Spelling Checker: Patient, Intervention, Comparison, Outcome. [acesso em 13 set 2016]. Disponível: https://pubmedhh.nlm.nih.gov/nlmd/pico/piconew.php.

17. Smith CA, Betts D. The practice of acupuncture and moxibustion to promote cephalic version for women with a breech presentation: implications for clinical practice and research. Complement Ther Med. [Internet] 2014;22(1) [acesso em 03 jan 2016]. Disponível: http://dx.doi.org/10.1016/j.ctim.2013.12.005.

18. Vas J, Aranda-Regules JM, Modesto M, Ramos-Monserrat M, Barón M, Aguilar I, et al. Using moxibustion in primary healthcare to correct non-vertex presentation: a multicentre randomised controlled trial. Acupunct Med. [Internet] 2013;31(1) [acesso em 05 jan 2016]. Disponível: http://dx.doi.org/10.1136/acupmed-2012-010261.

19. Guittier MJ, Pichon M, Dong H, Irion O, Boulvain M. Moxibustion for breech version: a randomized controlled trial. Obstet Gynecol. [Internet] 2009;114(5) [acesso em 10 jan 2016]. Disponível: http://dx.doi.org/10.1097/ AOG.0b013e3181bc707a.

20. Mitchell M, Allen K. An exploratory study of women's experiences and key stakeholders views of moxibustion for cephalic version in breech presentation. Complement Ther Clin Pract. [Internet] 2008;14(4) [acesso em 03 jan 
2016]. Disponível: http://dx.doi.org/10.1016/j.ctcp.2008.05.002.

21. Vas J, Aranda JM, Barón M, Perea-Milla E, Méndez C, Ramírez C, et al. Correcting non cephalic presentation with moxibustion: study protocol for a multi-centre randomised controlled trial in general practice. BMC Complement Altern Med. [Internet] 2008;(8) [acesso em 12 jan 2016]. Disponível: http://dx.doi.org/10.1186/14726882-8-22.

22. Cardini F, Lombardo P, Regalia AL, Regaldo G, Zanini A, Negri MG, et al. A randomised controlled trial of moxibustion for breech presentation. BJOG. [Internet] 2005;112(6) [acesso em 05 jan 2016]. Disponível: http:// dx.doi.org/10.1111/j.1471-0528.2005.00634.x.

23. Neri I, Airola G, Contu G, Allais G, Facchinetti F, Benedetto C. Acupuncture plus moxibustion to resolve breech presentation: a randomized controlled study. J Matern Fetal Neonatal Med. [Internet] 2004;15(4) [acesso em 12 jan 2016]. Disponível: http://dx.doi.org/10.1080/14767050410001668644.

24. Neri I, Fazzio M, Menghini S, Volpe A, Facchinetti F. Non-stress test changes during acupuncture plus moxibustion on BL67 point in breech presentation. J Soc Gynecol Investig. [Internet] 2002;9(3) [acesso em 05 jan 2016]. Disponível: http://citeseerx.ist.psu.edu/viewdoc/download?doi=10.1.1.849.6556\&rep=rep1\&type=pdf.

25. Cardini F, Weixin H. Moxibustion for correction of breech presentation: a randomized controlled trial. JAMA. [Internet] 1998;280(18) [acesso em 03 jan 2016]. Disponível: http://dx.doi.org/10.1001/jama.280.18.1580. 